EPJ Web of Conferences 16, 06013 (2011)

DOI: $10.1051 /$ epjconf/20111606013

(C) Owned by the authors, published by EDP Sciences, 2011

\title{
Mapping radio emitting-region on low-mass stars and brown dwarfs
}

\author{
S. Yu ${ }^{1, a}$, J.G. Doyle ${ }^{1}$, G. Hallinan ${ }^{2}$ and A.L. MacKinnon ${ }^{3}$ \\ ${ }^{1}$ Armagh Observatory, College Hill, Armagh BT61 9DG, N. Ireland \\ ${ }^{2}$ Centre for Astronomy, National University of Ireland, Galway, Ireland \\ ${ }^{3}$ The Department of Adult and Continuing Education, University of Glasgow, Scotland
}

\begin{abstract}
Strong magnetic activity in ultracool dwarfs (UCDs, spectral classes later than M7) have emerged from a number of radio observations, including the periodic beams. The highly (up to $100 \%$ ) circularly polarized nature of the emission point to an effective amplification mechanism of the high-frequency electromagnetic waves - the electron cyclotron maser (ECM) instability. Several anisotropic velocity distibution models of electrons, including the horseshoe distribution, ring shell distribution and the losscone distribution, are able to generate the ECM instability. A magnetic-field-aligned electric potential would play an significant role in the ECM process. We are developing a theoretical model in order to simulate ECM and apply this model to map the radio-emitting region on low-mass stars and brown dwarfs.
\end{abstract}

\section{INTRODUCTION}

A large number of recent observations indicate that intense magnetic activity exists in ultracool dwarfs which have spectral type later than M7, including some brown dwarfs with L, T type spectra. Berger et al. (2001) [4] reported the first detection of quiescent and flaring radio emission from the brown dwarf LP944-20, with anomalous quiescent radio luminosity larger than predicted from an empirical relation between the X-ray and radio luminosities of active stars with spectral types from F to M [6] by at least four orders of magnitude. Up to now, about 11 radio active ultracool dwarfs, with spectral type from M8 to L3.5, including a binary system, have been found from various survey $[1,3,7,8]$. Three of these have been shown to have periodic radio emission [7, 8]. Common features of the radio observation in these ultracool dwarfs are high brightness temperature and highly circular polarization emission, which suggest that the dominant radio emission mechanism in the ultracool dwarfs is the electron cyclotron maser (ECM) instability - direct wave magnification process of the free-space radiation modes [14]. This process could be induced by some kind of anisotropic velocity distributions of electrons, such as a loss-cone distribution, a ring shell distribution, or a horseshoe distribution.

Lau \& Chu (1983) [9] found that the ECM may be exited in a plasma with a velocity distribution of a loss-cone, i.e., $f_{\mathrm{i}} \sim p_{\perp}^{2 j} \exp \left(-p^{2} / \Delta p^{2}\right)$, of weakly relativistic electrons with the temperature exceeding a few tens of $\mathrm{keV}$, the appearance of the maser may depend on the plasma density. However, as discussed by Louarn et al. (1990) [10] based on Viking satellite observations, the loss-cone maser is insufficient to produce the auroral kilometric radiation (AKR) which may result from the another more effective velocity distribution of electrons. This suggestion is also supported by the result of Ergun et al. (2000) [5]. Pritchett (1984) [11] pointed out that a shell instability (or an incomplete shell instability) caused by a magnetic-field-aligned electric field would generate intense cyclotron emissions, in which a ring shell distribution, i.e., $f_{\mathrm{i}} \sim \frac{1}{p_{\perp}} \delta\left(p_{\perp}-p_{\mathrm{r}}\right) \delta\left(p_{\|}\right)$( $p_{r}$ is the ring momentum), of electrons is required. In such a

\footnotetext{
ae-mail: syu@arm.ac.uk
}

This is an Open Access article distributed under the terms of the Creative Commons Attribution-Noncommercial License 3.0, which permits unrestricted use, distribution, and reproduction in any noncommercial medium, provided the original work is properly cited. 


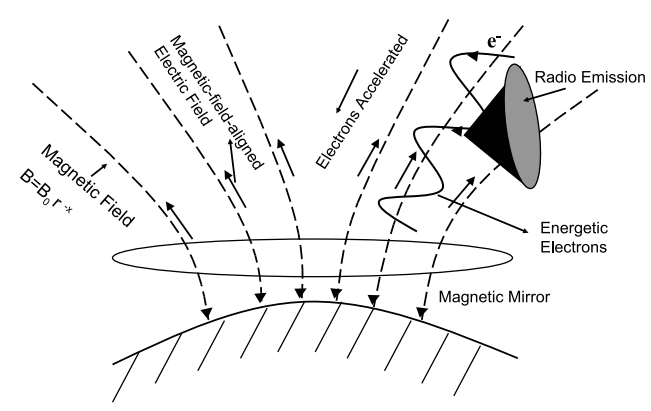

Figure 1. A model for coherent radio emission from ultracool dwarfs.

model, the initial electron-distribution function would be deformed by the presence of a magnetic-fieldaligned electric field which may accelerate the down-going electrons to a relativistic state and the mirror effect of the magnetic field which may increase the pitch angle of the electrons, so that an incomplete ring-shell distribution namely the so-called horseshoe distribution would be formed. We are developing a theoretical model to simulate the ECM instability and apply this model to map the emitting-region on ultracool dwarfs ${ }^{1}$. In this paper, we describe the method and preliminary results.

\section{SIMULATION MODEL}

We consider a simple one-dimensional model in which a group of precipitating electrons move along the variable magnetic field that depends on the height to the surface of an ultracool dwarf (Fig. 1). We assume this magnetic field in the form of

$$
B(r)=B_{0}\left(\frac{r}{R_{0}}\right)^{-2}, \quad r \geq R_{0}
$$

where $B_{0}$ is magnetic field strength of the surface of an ultracool dwarf, $R_{0}$ is the radius of the ultracool dwarf, and $r$ is the height of electrons to the surface of the ultracool dwarf.

The electrons and electromagnetic field should be satisfied with Maxwell's equations, Poisson's equation and motion equation. We assume that the radio wave produced by ECM can escape from plasma in the magnetosphere of ultracool dwarf, so the cyclotron frequency should satisfy with a requirement of $v_{\mathrm{p}} / \nu_{\mathrm{c}}<0.4$ (for observation, $<0.1$ ), where $v_{\mathrm{c}} \approx 2.86 \times B \mathrm{MHz}$ is cyclotron frequency and $v_{\mathrm{p}} \approx 9 \times 10^{-3}\left(n_{\mathrm{e}} \mathrm{cm}^{-3}\right)^{1 / 2} \mathrm{MHz}$ is plasma frequency. From the requirement, we can infer that the number density of electrons should $<10^{10} \mathrm{~cm}^{-3}$.

We adopt different initial velocity distribution of electrons, such as a $\kappa$-distribution, $f_{\mathrm{i}}(v) \propto[1+$ $\left.\frac{v^{2}}{\kappa v_{\mathrm{e}}^{2}}\right]^{-\kappa-1}$, a simple power law distribution, $f_{\mathrm{i}}(v) \propto v^{-\alpha}$. These different distribution would have an effect on the radio emission.

\section{PRELIMINARY RESULTS}

Fig. 2 shows a ring shell distribution (right panel) and a deformed ring shell distribution (horseshoe distribution, left panel). Taking into account flux $\mathbf{S}_{0}=\frac{c}{4 \pi}\left|\mathbf{E}^{2}\right| \mathbf{n}$, we can obtain a relation between flux and frequency as in Fig. 3. In this case, we assume an electron number density of $1 \times 10^{9} \mathrm{~cm}^{-3}$, a simple power law velocity distribution $f_{\mathrm{i}} \propto v^{-2}$, the size of emission region of $100 \mathrm{~km} \times 100 \mathrm{~km} \times 1 \mathrm{~km}$, and

\footnotetext{
${ }^{1}$ The radio emission from M8.5V dwarf TVLM 513-46546 can be represented by its rotation and the ECM instability [15].
} 


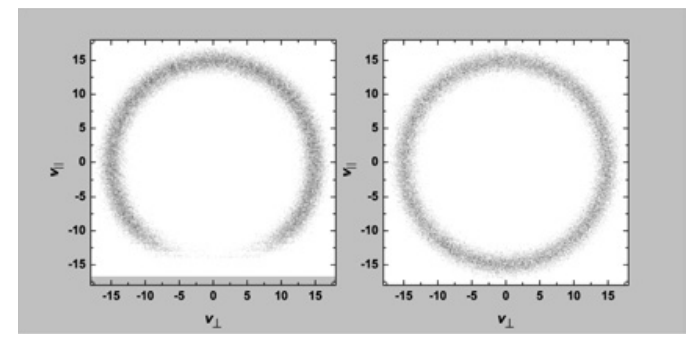

Figure 2. A ring shell distribution and a deformed ring shell (horseshoe) distribution.

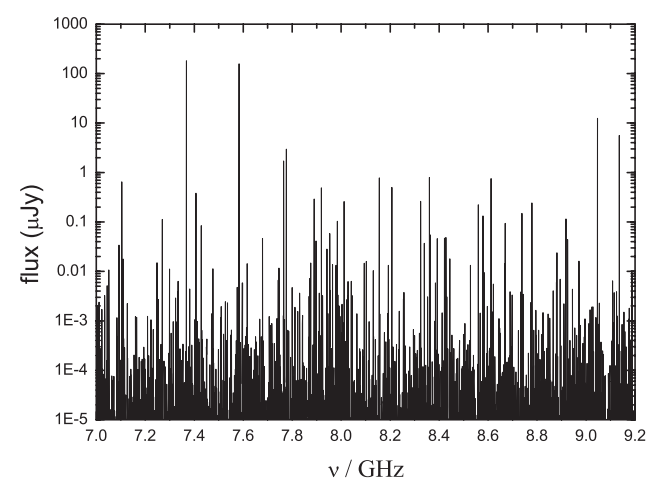

Figure 3. A simple example (flux versus frequency) for the coherent radio emission from an ultracool dwarf.

the distance from the ultradwarf to us of $10 \mathrm{pc}$. Note that if we consider a ECM growth rate $\Gamma$, the flux would be $\mathbf{S}=\mathbf{S}_{0} \Gamma^{4}$, since $\mathbf{E} \propto \Gamma^{2}$.

\section{DISCUSSION}

As an effective wave amplification mechanism, the ECM instability might be involved in many radio emission processes. Winglee \& Dulk (1986) [13] found that the ECM instability may induce both solar continuum radio emission of type IV and $\mathrm{V}$ by producing a positive $\partial f / \partial p_{\perp}$ to drive the instability. The results of Aschwanden (1990) [2] suggested that solar radio millisecond spikes are associated with the saturation of the ECM instability. The ECM was also applied to radio emissions from binary systems, and narrowband emission from dwarf $\mathrm{M}$ flare stars. The strong radio emission from some planets, such as AKR, DAM, HOM and SKR, are also attributed to the ECM instability $[12,16]$.

These results indicate that the ECM instability is a prevalent radio emission mechanism from different objects, from low-mass stars like the Sun to some small planets like the Earth. By comparing the model with the observations, we should be able to obtain a clear understanding on the topology structure and formation of the magnetic field, the number and energy density distribution of the electrons, and the interaction between the electrons and ambient electromagnetic field.

\section{References}

[1] Antonova, A., et al., A\&A, 487, (2008) 317

[2] Aschwanden, M. J., A\&A, 237, (1990) 512

[3] Berger, E., ApJ, 648, (2006) 629

[4] Berger, E., et al., Nature, 410, (2001) 338 
[5] Ergun, R. E., et al., ApJ, 538, (2000) 456

[6] Güdel, M., Benz, A. O., ApJ, 405, (1993) L63

[7] Hallinan, G., et al., ApJ, 663, (2007) L25

[8] Hallinan, G., et al., ApJ, 684, (2008) 644

[9] Lau, Y. Y., Chu, K. R., Phys Rev Lett, 50, (1983) 243

[10] Louarn P., et al., J. Geophys. Res., 95, (1990) 5983

[11] Pritchett, P. L., J. Geophys. Res., 89, (1984) 8957

[12] Treumann, R. A., A\&ARv, 13, (2006) 229

[13] Winglee, R. M., Dulk, G. A., ApJ, 310, (1986) 432

[14] Wu, C. S., Lee, L. C., ApJ, 230, (1979) 621

[15] Yu, S., et al., A\&A, 525, (2011) A39

[16] Zarka, P., Adv. Space Res., 12(8), (1992) 99 